\title{
Os impactos da difusão tecnológica na bovinocultura leiteira: um estudo dos integrantes da cadeia agroindustrial do leite em um município de Minas Gerais
}

\author{
The impacts of technology dissemination in dairy cattle: a study of milk \\ agribusiness chain in a Minas Gerais' county, Brazil
}

\author{
Henrique Cordeiro Martins ${ }^{\mathrm{I}}$ Cristiana Fernandes De Muylder ${ }^{\mathrm{I}^{*}}$ \\ Célio Adriano Lopes ${ }^{I I}$ Jefferson La Falce ${ }^{\mathrm{I}}$
}

\section{RESUMO}

\begin{abstract}
O objetivo deste artigo foi analisar a inserção de tecnologia na cadeia agroindustrial do leite no município de Patos de Minas, no Estado de Minas Gerais. Foi realizado um estudo descritivo, de natureza quantitativa, com três segmentos pertencentes à cadeia agroindustrial de leite do município, constituídos por trinta produtores rurais, duas associações de produtores e duas indústrias processadoras de leite, totalizando 34 questionários aplicados aos três segmentos identificados. Os resultados demonstraram que, no período investigado, o setor de bovinocultura leiteira no município passou por profundas mudanças estruturais no que tange a difusão tecnológica, a inovação e a logística, ocasionando, dentre outros, a diminuição do número de empresas rurais, melhoria da infraestrutura e aumento de certificações ambientais. Portanto, pode-se observar que o ambiente mudou e que a incorporação de inovações tecnológicas por parte dos segmentos pesquisados, bem como a modernização de infraestrutura focaram melhoria na produção e processamento do leite.
\end{abstract}

Palavras-chave: cadeia agroindustrial do leite, inovação, difusão tecnológica.

\section{ABSTRACT}

The paper purpose was to examine the technology inclusion of the milk agro-industrial chain in the city of Patos de Minas, in Minas Gerais. It was a descriptive and quantitative research using three business segments. The research involved thirty farmers, two producers associations and two milk processing industries, totaling 34 interviews. The results showed that, in the investigated period, the sector suffered significant structural changes regarding technological diffusion, innovation and logistics, resulting in, among others, a decrease in the number of rural enterprises, improving infrastructure and increasing environmental certifications. Therefore, it can be seen that the environment has changed and that the incorporation of technological innovations by the surveyed sectors, as well as upgrading of the infrastructure have focused on improving the production and processing of milk.

Key words: milk agro-industrialchain, innovation, technology dissemination.

\section{INTRODUÇÃO}

O Sistema Agroindustrial de leite (SAG lácteo) do país contribui significativamente para a elevação do Produto Interno Bruto (PIB). Nesse sentido, a partir da expansão da economia brasileira na década de 1990, o SAG lácteo se estruturou para adequar-se à competição do mercado no cenário interno, como também no mercado externo, em função, principalmente, da inserção de empresas multinacionais no país. Essa reestruturação influenciou na adequação do empreendimento rural tradicional, que sofreu mudanças de especialização da mão de obra. O resultado dessas mudanças tidas como gerenciais e produtivas nas propriedades rurais especializadas foi percebido, principalmente, por meio da redução dos custos de produção, do aumento da competitividade e da incorporação de inovações tecnológicas e gerenciais, tornando o empreendimento rural mais lucrativo, mais eficiente e mais competitivo.

Algumas estratégias se fazem pertinentes para prover aos produtores e trabalhadores da zona rural conhecimentos sobre seus direitos e deveres.

'Programa de Pós-graduação em Administração (PPGA), Universidade Fundação Mineira de Educação e Cultura (FUMEC), 30130-009, Belo Horizonte, MG, Brasil. E-mail: cristiana.muylder@fumec.br. *Autor para correspondência.

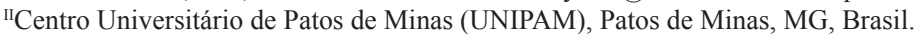


Nesse contexto, o gestor pode atuar como agente colaborador e impulsionador de reformas no processo de inovação, contribuindo para melhoria da qualidade de vida da população do meio rural, principalmente, aos vinculados ao setor de bovinocultura. Dessa forma, elabora-se o questionamento da pesquisa pautado no seguinte problema de investigação: quais estratégias de inovação tecnológica foram utilizadas pelas empresas da Cadeia Agroindustrial do Leite no município de Patos de Minas-Minas Gerais e quais os principais dificultadores desta incorporação?

Nesse sentido, o objetivo desta pesquisa foi analisar a inserção de tecnologia na cadeia agroindustrial do leite no município de Patos de Minas, no período de 1990 a 2009, verificando quais as estratégias de inovação foram utilizadas pelas empresas, bem como os fatores dificultadores na incorporação das inovações tecnológicas.

A pesquisa é importante porque analisa fatores decorrentes da incorporação e uso de tecnologias no setor lácteo o que pode contribuir para a preservação de valores regionais. Além disso, este estudo impulsiona discussões acadêmicas relacionadas à gestão da cadeia agroindustrial do leite nos aspectos político, econômico e tecnológico, tendo em vista o aperfeiçoamento da cadeia produtiva, viabilizando a produção e comercialização de derivados do leite, conforme a demanda e as exigências do mercado. A escolha da região de Patos de Minas se deu por critério de acessibilidade por parte dos pesquisadores, visto que o ambiente agropecuário requer contatos pessoais e conhecimento da região.

De acordo com NANTES \& SCARPELLI (2007), os empreendimentos rurais são classificados em três tipos: (i) empreendimentos rurais tradicionais; (ii) empreendimentos rurais em transição; (iii) empreendimentos rurais modernos. Os empreendimentos rurais tradicionais são segmentos que apresentam estruturas organizacionais baseadas no modelo familiar. Os rurais modernos são tidos como produtores de escala, característicos do modelo patronal. No modelo familiar, os produtores escoam a produção diretamente para os consumidores via venda no segmento varejo, ou escoam para a associação ou cooperativa, mediante relação por cota de participação. No modelo patronal, com estrutura mais empreendedora, o produtor em escala consegue enviar a produção para o mercado varejo numa relação direta ou para a agroindústria através de relação contratual.

O Manual de Oslo (OCDE, 2005) lista fatores relacionados aos efeitos de inovação, que servem de base para verificar se os objetivos relacionados à ela foram alcançados. Os efeitos e objetivos de inovação passíveis de investigação estão distribuídos em quatro grupos referentes: (1) à empresa (competição, demanda e mercado), (2) à produção e distribuição, (3) à organização do local de trabalho e (4) outros. Esses quatros grupos compreendem o conjunto de tópicos que são avaliados com base nas quatro áreas de inovações já citadas e constantes do Manual de Oslo. Dessa forma, o uso desses dados pode facilitar a avaliação das formas de inovação presentes em uma empresa (OCDE, 2005).

No entanto, há fatores que dificultam as atividades de inovação nas empresas, o Manual de Oslo (OCDE, 2005) lista os principais fatores limitadores de inovações - (1) fatores relativos ao custo; (2) fatores relativos aos conhecimentos; (3) fatores de mercado; fatores institucionais; (4) outras razões para não inovar -, em que cada tópico contido nos grupos está relacionado com as quatro áreas de inovação, a saber: inovação de produto, inovação no processo, inovação organizacional e inovação de marketing.

\section{MATERIAL E MÉTODOS}

Foi realizada uma pesquisa de natureza quantitativa, do tipo descritiva, por meio de um estudo de caso de uma cadeia agroindustrial do leite no município de Patos de Minas/MG, realizada sob a percepção de três agentes: 1) percepção produtor rural, de acordo com a classificação proposta por NANTES \& SCARPELLI (2007): modelo patronal e modelo familiar. A seleção dos respondentes foi realizada com base em amostra não probalística por acessibilidade e tipicidade; 2) percepção gestores de duas associações de produtores rurais; 3) percepção de uma indústria de processamento de leite.

$\mathrm{O}$ estudo de caso se refere à cadeia agroindustrial do leite no município de Patos de Minas/MG, especificamente, nos segmentos de produção primária (produtores rurais), captação (associações de tanques comunitários) e indústria processadora (indústrias de produtos lácteos). O instrumento de coleta de dados foi constituído por questionário, direcionado aos participantes da investigação. A amostra (Tabela 1) foi definida por tipicidade e acessibilidade, considerando que um dos pesquisadores reside em Patos de Minas e é administrador da Fazenda Experimental, do Campus avançado da Faculdade de Ciências Agrárias e Engenharia do Centro Universitário de Patos de Minas. Para o tratamento dos dados, foram utilizados os recursos da estatística descritiva para 
Tabela 1 - Descrição da distribuição do questionário.

\begin{tabular}{lllll}
\hline Tipo & Organizaçao & (n) & Função & Instrumento \\
\hline Empresa rural & Modelo patronal & 20 & Produtor rural & Questionário \\
& Modelo familiar & 10 & Produtor rural & Questionário \\
Entidade Coletiva & Associação de produtores & 02 & Presidente & Questionário \\
Indústria processadora & láctea & 02 & Gerente & Questionário \\
& & & Gerente & \\
\hline
\end{tabular}

Fonte: Dados da pesquisa.

fazer as análises pertinentes, a saber: medidas de posição, limitada às medidas de tendência: média ( $\mathrm{X})$, moda (X_o) e mediana ( $\left.\mathrm{X}^{\sim}\right)$, além de medidas de dispersão, por possibilitarem "avaliar o grau de confiabilidade de uma medida de tendência central numa amostra como parâmetro da população", conforme afirma MATTAR (1996,). O questionário continha duas partes sobre a difusão tecnológica. $\mathrm{Na}$ primeira parte, o objetivo foi verificar quais estratégias de inovação eram utilizadas pelas empresas nos últimos cinco anos.

Já na segunda parte, avaliaram-se dois fatores que podem dificultar às empresas incorporarem inovações tecnológicas. Os fatores avaliados foram: Fator de Mercado e Fator Institucional. Os Fatores de Mercado são constituídos por variáveis representadas pelos itens de respostas "a" e "b": a) demanda incerta para bens ou serviços inovadores; b) mercado potencial dominado pelas empresas estabelecidas. Os Fatores Institucionais são constituídos por variáveis representadas pelos itens de respostas de "c" a "g", a saber: c) carência de infraestrutura; d) fragilidade dos direitos de propriedade; e) legislação, regulações, padrões, tributação, f) outras razões para não inovar (não necessidade de inovar decorrente de inovações antigas); g) outras razões para não inovar (não necessidade decorrente da falta de demanda por inovações). Esta etapa do questionário era composta de uma escala do tipo likert de cinco pontos que variava de 1- "existe totalmente" a 5 -"não existe totalmente".

\section{RESULTADOS E DISCUSSÃO}

Dos produtores rurais

No primeiro questionamento, com base noVER 2, os produtores rurais não avaliaram os itens de respostas 11, 13, 14 e 16. Porém, entre as estratégias avaliadas, registraram-se quatro itens de respostas: 3) cursos e treinamento gerencial; 8) consultorias externas; 9) associação de classe; 15) implantação de soluções de logísticas.
No que se refere a cursos e treinamento gerencial, pode-se indicar que os produtores rurais (modelo patronal) estão comprometidos com a qualificação profissional. As consultorias externas mostram que os produtores rurais tanto do modelo patronal como do familiar buscam orientações externas. A associação de classe indica que os produtores rurais têm consciência quanto à importância da união para a busca de soluções para problemas reais. Portanto, os dados indicam que os produtores rurais nos dois modelos de gestão, patronal ou familiar, têm consciência quanto à incorporação de inovações tecnológicas e organizacionais para a melhoria do processo produtivo o que de acordo com TIGRE (2006), trata de uma "estratégia de imitação", haja vista que as empresas brasileiras investem pouco em inovação no campo de P\&D.

$\mathrm{Na}$ segunda questão do questionário, fatores de mercado, variável $b$ "Mercado potencial dominado pelas empresas estabelecidas", do modelo patronal, observa-se que os dados estão dispersos, apresentando variação entre $51,64 \%$ a $69,28 \%$, e que as respostas se concentraram nas categorias "não existe parcialmente / não existe totalmente.

Tal evidência parece indicar que os produtores rurais conhecem a realidade do mercado em que estão inseridos, já que as médias e grandes empresas dominam o mercado lácteo tanto no segmento produtivo, como no de processamento de leite e derivados. Este aspecto, de acordo com TIGRE (2006), trata-se de um ponto negativo referente ao impacto de difusão de tecnologia do ponto de vista econômico e social, pois o número de empresas tende a aumentar, chegando a comprometer a permanência das pequenas empresas no mercado.

No modelo familiar, constata-se que os dados estão dispersos em relação às respectivas médias, apresentando variação entre 40,0\% a 69,28\%, e que as respostas se concentraram nas categorias "existe totalmente / existe parcialmente". Os dados indicam ser condizente com a realidade dos produtores 
rurais de modelo familiar, porque impacta a dinâmica das empresas e contribui para a diminuição do número de empresas. De acordo com TIGRE (2006), tal evidência indica ser um ponto negativo referente ao impacto de difusão de tecnologia do ponto de vista econômico e social.

Já em relação aos fatores institucionais, o modelo patronal apresenta médias das variáveis iguais (= 5), sendo a variável "e" (Legislação, regulações, padrões, tributação), com menor dispersão de respostas (Variância -Var $=4,00$ ); Desvio padrão - $\mathrm{dp}=2,00 \mathrm{e}$ Coeficiente de Variação - CV $=40,0 \%$ ). Já em relação às categorias de respostas, as variáveis "ff, g" (Outras razões para não inovar -inovações antigas; Outras razões para não inovar-falta de demanda) têm os dados concentrados nos itens "existe totalmente/existe parcialmente", enquanto as variáveis " $\mathrm{d}$, e" (Fragilidade dos direitos de propriedade; Legislação, regulações, padrões, tributação) os dados estão concentrados nos itens "não existe parcialmente / não existe totalmente".

$\mathrm{Na}$ variável "c" (Carência de infraestrutura), os dados indicam que parte dos produtores rurais persiste em inovações antigas. Possivelmente, este fato pode ser resultante da falta de recursos financeiros necessários à aquisição de novas tecnologias. No entanto, se as empresas não inovam porque não há demanda por inovações (variável “g”), como elas se adequam com relação às exigências do mercado, dos consumidores e das legislações.

No modelo familiar, as variáveis "f, g” apresentaram valores próximos à média do grupo (=5), com desvios padrão $(1,41)$ e $\mathrm{CV}$ de $28,28 \%$, com categorias de respostas concentradas no item "não existe parcialmente". Por outro lado, as variáveis "c, e" têm as respostas concentradas nos itens "não existe parcialmente / não existe totalmente".

Diante dessas evidências, observa-se que as variáveis "c" e "e" são as que estão mais próximas da realidade dos produtores familiares, pois a carência de infraestrutura (variável "c") e a forte presença da legislação, tributação (variável "e") sobre o processo produtivo dificultam o dia a dia do produtor familiar. No primeiro caso, há fonte de financiamento da produção agrícola - por exemplo, o Pronaf. No entanto, de acordo com NANTES \& SCARPELLI (2007), o acesso ao crédito pelo produtor rural nas instituições financeiras indica ser um fator limitante, devido aos riscos inerentes à produção rural.

Das associações comunitárias

No primeiro questionamento, as associações comunitárias pesquisadas não avaliaram os itens de resposta 7, 11, 13, 14 e 15 (QUADRO 2), pois as categorias não envolvem diretamente as associações em relação à função que desempenham na cadeia produtiva, conforme pode ser constatado nos estudos de GOMES \& LEITE (2001) e NANTES \& SCARPELLI (2007).

Observa-se que as inovações sinalizadas pelos representantes das duas associações se concentraram no tipo Inovações tecnológicas (TIGRE, 2006), categorias de 1 a 11, em que as duas associações citaram 11 fontes de inovação juntas. Já para o grupo do tipo Inovações Organizacionais, categorias de 12 a 16, houve duas indicações, categorias 12 e 16 .

Constata-se que, na Associação 1, houve 9 indicações de incorporação de Inovações tecnológicas, sendo que 7 delas correspondem a 50\% das respostas das categorias de 2 a 6, 8 e 9; e dois que corresponderam a $100 \%$ das respostas das categorias 1 e 10. Na Associação 2, houve sete indicações de incorporação de Inovações tecnológicas, sendo que todas correspondem a $50 \%$ das respostas das categorias 2 a 6,8 e 9 .

Os dados descritos revelam que as duas Associações procuraram incorporar inovações tecnológicas e, em menor escala, inovações organizacionais. Essa postura permite às Associações modernizarem a estrutura gerencial e a operacional, adequando-se, portanto, às mudanças que ocorrem no setor, conforme sinalizado por NANTES \& SCARPELLI (2007).

No segundo questionamento sobre Fatores de Mercado, os dados das duas Associações são divergentes, pois a Associação 1 considerou que as variáveis - "a", "b" (Demanda incerta para bens ou serviços inovadores; Mercado potencial dominado pelas empresas estabelecidas) "não existe parcialmente" na região, enquanto a Associação 2 considerou que as mesmas variáveis "existe parcialmente". No entanto, considerando os estudos realizados por TIGRE (2006), é possível observar que a avaliação da Associação 1 indica que está distante da realidade em que está inserida, principalmente com relação ao aumento do processo de fusões, aquisições e alianças estratégicas realizadas por médias e grandes empresas no setor, conforme sinalizado também por NANTES \& SCARPELLI, (2007).

Já em relação aos Fatores Institucionais, os dados descritos pelos representantes das duas associações indicam que, para a Associação 1, as variáveis "c", "f", "g" (Carência de infraestrutura; Outras razões para não inovar-inovações antigas; Outras razões para não inovar-falta de demanda) 
foram avaliadas como "existe parcialmente", enquanto para as variáveis "d", "e" (Fragilidade dos direitos de propriedade; Legislação, regulações, padrões, tributação), como "não existe parcialmente". Já a Associação 2 avaliou todas as variáveis como "não existe parcialmente".

A partir dos dados apresentados, constatase que as respostas dadas às variáveis "d", "e" foram comuns, pois a questão de direitos de propriedade no aspecto fiscalização precisa ser mais bem trabalhada pelas autoridades responsáveis, para que sejam evitados danos financeiros e econômicos às empresas detentoras de marcas e patentes. No aspecto, legislação-regulações-padrões-tributação, destacam-se como pontos positivos o processo de desregulamentação do setor, iniciado a partir de 1991, e a abertura da economia do País para o mercado internacional (GOMES \& LEITE, 2001), assim como o aumento de certificações e regulamentos, sob o ponto de vista ambiental, conforme estudos de TIGRE (2006).

Das indústrias processadoras

No primeiro questionamento, os representantes das indústrias processadoras, 1 gerente não avaliou as categorias de respostas 11 e 13, possivelmente por não se relacionar diretamente com as atividades de rotina das empresas investigadas.

Com base nos dados levantados, observase que as indústrias processadoras pesquisadas incorporam 90,9\% das inovações listadas no grupo Inovações tecnológicas e $80 \%$ das inovações listadas no grupo Inovações organizacionais nos últimos cinco anos.

A partir dos resultados descritos, constata-se que a empresa pesquisada demonstra ter consciência da importância de incorporar inovações tecnológicas e organizacionais, o que sugere aumento da qualidade dos bens e serviços. Portanto, relacionase ao efeito da inovação incorporada no processo produtivo em relação a inovações de produto, processo e organizacionais, de acordo com o Manual da OCDE, "Fatores relacionados aos objetivos e efeitos da inovação" (OCDE, 2005).

No segundo questionamento referente aos Fatores de Mercado, os dados avaliados pelo respondente da indústria processadora considerou as variáveis "a, b" (Demanda incerta para bens ou serviços inovadores; Mercado potencial dominado pelas empresas estabelecidas) como "existe parcialmente" na região. Em relação à variável "a", a cadeia agroindustrial do leite procura de adequar às mudanças que ocorrem no setor, principalmente com a forte influência exercida pelo processo de globalização da economia (GOMES \& LEITE, 2001). Com relação à variável "b", e ancorado nos estudos de TIGRE (2006) sobre os impactos da difusão tecnológica, constata-se que, sob o ponto de vista econômico, há concentração de indústrias no setor, vindo a impulsionar o aumento da escala de produção.

Já em relação aos fatores institucionais, as variáveis “c, d, e" (Carência de infraestrutura; Fragilidade dos direitos de propriedade; Legislação, regulações, padrões, tributação) foram avaliadas pelo respondente da indústria como "existe parcialmente". Não houve indicação de respostas para as variáveis "f, g" (Outras razões para não inovar-inovações antigas; Outras razões para não inovar-falta de demanda). Observa-se, de acordo com os dados, que há carência de infraestrutura, variável "c", porém, é uma questão que precisa ser melhor investigada mediante estudos futuros, a fim de verificar até que ponto os fatores institucionais tornam-se empecilhos para o desenvolvimento da empresa pesquisada.

Já com relação à variável "e" Legislação, regulações, padrões, tributação - a resposta dada pelo respondente parece ser favorável à indústria pesquisada, pois a desregulamentação do setor, a partir de 1991, permitiu maior liberdade no mercado em decorrência da desregulamentação do setor lácteo (GOMES \& LEITE, 2001).

\section{CONCLUSÃO}

Especificamente sobre o setor lácteo brasileiro pode-se dizer que a abertura do mercado, a partir de 1991, causou desregulamentação do mercado de leite, abertura da economia para o mercado internacional e estabilização de preços com a implantação do Plano Real. Essas mudanças propiciaram o aumento da produção de leite, principalmente, com a expansão do setor para a região do cerrado e em regiões do Triângulo Mineiro e Alto Paranaíba .

Diante disso, a incorporação de inovações tecnológicas e gerenciais tornou-se fator primordial para a permanência dos agentes da cadeia agroindustrial do leite, porque as transformações impulsionam o aumento da produtividade, a redução do número de empreendimentos rurais de base familiar, e, consequentemente, a redução do preço do leite e o aumento da especialização da mão de obra rural, metamorfoseando, portanto, empreendimentos rurais tradicionais em empreendimentos lucrativos, eficientes e competitivos. 
Com os dados analisados e discutidos, chegou-se à conclusão de que os produtores rurais são conscientes quanto à incorporação de inovações tecnológicas e organizacionais para a melhoria do processo produtivo em suas propriedades e na própria cadeia agroindustrial do leite (aquisição de fontes externas de tecnologia - por exemplo, de máquinas e equipamentos).

Todavia, no processo de difusão tecnológica, Fatores de Mercado, os produtores são conscientes de que o aumento de empresas de médio e grande porte, sob o ponto de vista econômico e social compromete a permanência deles no mercado. No aspecto Fatores Institucionais, há persistência de inovações antigas, podendo ser resultante da falta de recursos financeiros. Incide também sobre eles a carência de infraestrutura, mesmo havendo fonte de financiamento como o Pronaf, bem como há forte presença de legislação e tributos, dificultando a permanência no mercado.

As associações comunitárias incorporaram, como estratégias organizacionais, em maior escala, inovações tecnológicas, e, em menor escala, inovações organizacionais, adequando-se, portanto, às mudanças do mercado. Em relação aos Fatores de Mercado, Fragilidade dos direitos de propriedade, há necessidade de que sejam melhor fiscalizados pelas autoridades responsáveis, a fim de evitar danos financeiros e econômicos às empresas detentoras de marcas e patentes. Já em relação ao item Legislação, regulações, padrões, tributação, este foi avaliado como positivo, pois permitiu a desregulamentação do setor a partir de 1991 com a abertura da economia, bem como, aumentou as certificações e regulamento no aspecto ambiental.

As indústrias processadoras adotaram, como estratégia organizacional, a incorporação de inovações tecnológicas e gerenciais, podendo resultar no aumento da qualidade dos produtos dispostos no mercado lácteo. Sobre o processo de difusão tecnológica, em relação aos Fatores de Mercado, as empresas procuram se adequar ao mercado, porém, no item Mercado Potencial, dominado pelas empresas estabelecidas, as empresas desconhecem a incidência deste na região. Já em relação aos Fatores Institucionais, Carência de infraestrutura, as empresas sinalizaram que essas características existem entre elas, todavia, é uma questão que precisa ser melhor investigada, a fim de verificar até que ponto os fatores institucionais se apresentaram como limitadores à modernização do parque industrial das empresas.

Percebe-se que os dados aferidos na pesquisa mostram a evolução por que passou o setor de bovinocultura leiteira e as profundas mudanças estruturais no que tange à tecnologia, inovação e logística, entre outros itens da cadeia agroindustrial do leite na região do município de Patos de Minas.

\section{AGRADECIMENTOS}

Financiamento Fundação de Amparo à Pesquisa do estado de Minas Gerais (FAPEMIG), Coordenação de Aperfeiçoamento de Pessoal de Nível Superior (CAPES) e Conselho Nacional de Desenvolvimento Científico e Tecnológico (CNPq).

\section{REFERÊNCIAS}

GOMES, A.T.; LEITE, J.L.B. O relacionamento na cadeia agroindustrial do leite para os novos tempos. In: GOMES, A.T. et al. (Ed.). O agronegócio do leite no Brasil. Juiz de Fora/MG: Embrapa Gado de Leite, 2001. Cap.10, p.139-165.

INSTITUTO BRASILEIRO DE GEOGRAFIA E ESTATÍSTICA (IBGE). Dados gerais municípios de Patos de Minas - 314800 (2007). Disponível em: < http://www.ibge.gov.br/cidadesat/ topwindow.htm?1>. Acesso em: 10 out. 2008.

MATTAR, F.N. Pesquisa de marketing. Edição Compacta. São Paulo: Atlas, 1996. 275 p.

NANTES, J.F.D.; SCARPELLI, M. Gestão da produção rural no agronegócio. In: BATALHA, M.O. (Coord.). Gestão agroindustrial. 3.ed. São Paulo: Atlas, 2007. Cap.10, p.556-584.

ORGANIZAÇÃO PARA A COOPERAÇÃO E DESENVOLVIMENTO ECONÔMICO (OCDE). Manual de Oslo: proposta de diretrizes para coleta e interpretação de dados sobre inovação tecnológica. Tradução de Flávia Gouveia. 3.ed. Brasília/DF: FINEP / OCDE, 2005. Disponível em: <http:// www.fiepr.org.br/inovaparana/uploadAddress/Manual $\% 20 \mathrm{de} \% 20$ Oslo\%20Terceira\%20Edi].pdf>. Acesso em: 05 dez. 2008.

TIGRE, P.B. Gestão da inovação: a economia da tecnologia no Brasil. Rio de Janeiro: Campus, 2006. 282 p. 